\title{
Decay and return of internal solitary waves with rotation
}

\author{
Karl R Helfrich* \\ Department of Physical Oceanography \\ Woods Hole Oceanographic Institution \\ Woods Hole, MA 02543 USA
}

(Dated: December 18, 2006)

\begin{abstract}
The effect of rotation on the propagation of internal solitary waves is examined. Wave evolution is followed using a new rotating extension of a fully-nonlinear, weakly nonhydrostatic theory for waves in a two-layer system. When a solitary wave solution of the non-rotating equations is used as the initial condition the wave initially decays by radiation of longer inertia-gravity waves. The radiated inertia-gravity wave always steepens, leading to the formation a secondary solitary-like wave. This decay and re-emergence process then repeats. Eventually a nearly localized wavepacket emerges. It consists of a longwave envelope and shorter, faster solitary-like waves that propagate through the envelope. The radiation from this mature state is very weak, leading to a robust, long-lived structure that may contain as much as $50 \%$ of the energy in the initial solitary wave. Interacting packets may either pass through one another, or merge to form a longer packet. The packets appear to be modulated, fully-nonlinear versions of the steadily translating quasi-cnoidal waves.
\end{abstract}

PACS numbers: Valid PACS appear here

Keywords: nonlinear internal waves, solitary waves, rotation

${ }^{*}$ Electronic address: khelfrich@whoi.edu 


\section{INTRODUCTION}

Long nonlinear internal solitary waves have received much attention over the last several decades due to the both in situ and remote sensing evidence for their common occurrence in coastal and marginal seas. During the same period, there have been great advances in understanding the dynamics of these waves. The canonical model for describing their dynamics is Korteweg-de Vries $(\mathrm{KdV})$ theory in which a balance between weak nonlinearity, represented by $\alpha=a / h_{s} \ll 1$, and weak nonhydrostatic dispersion, $\beta=\left(h_{s} / l\right)^{2}=O(\alpha)$, gives rise to the solitary waves. Here $a$ is a wave amplitude scale, $h_{s}$ is a depth scale, and $l$ is a wave lengthscale. KdV models with extensions to include bottom topography, dissipation, mean flows, and other effects have been applied to oceanographic and similar atmospheric observations with generally good results. The phenomenology, if not always the precise quantitative characteristics, is captured by the KdV class of theories (see reviews ${ }^{1,2}$ ).

One effect that has received somewhat less attention is that of the earth's rotation. This is because that while the waves are long with respect to the depth, they are still short compared to the internal deformation radius such that $\gamma=l / L_{R} \ll 1$. Here $L_{R}=c_{0} / f$ is the deformation radius, $c_{0}$ is the linear long wave phase speed, and $f$ is the Coriolis frequency. As a consequence, rotational effects are frequently ignored. However, if $\gamma^{2}=O(\alpha)$ then these weak rotational effects are comparable to the nonlinear and nonhydrostatic effects. For propagation in the $x$-direction in a domain unbounded in the transverse $y$-direction, the rotationally-modified $\mathrm{KdV}$, or Ostrovsky, equation ${ }^{3,4}$,

$$
\left(\eta_{t}+\alpha_{1} \eta \eta_{\xi}+\beta_{1} \eta_{\xi \xi \xi}\right)_{\xi}=\gamma_{1} \eta
$$

governs the evolution of the interfacial displacement $\eta(\xi, t)$. Here $t$ is time, and $\xi=x-$ $c_{0} t$. The coefficients $\alpha_{1}, \beta_{1}$, and $\gamma_{1}$ are functions of the vertical structure function (i.e., stratification $)^{4,5}$.

In the hydrostatic limit $\beta_{1}=0,(1)$ has periodic nonlinear inertia-gravity wave solutions (wavelengths of order $L_{R}$ ) in which the nonlinearity is balanced by the low-frequency rotational dispersion ${ }^{3,6}$. These periodic solutions exist up to a limiting amplitude, beyond which the rotational dispersion is unable to balance nonlinearity. At the limiting amplitude the solution is a sequence of parabolic arcs. For $\beta_{1}$ finite and $\gamma_{1}=0$, (1) reduces to the $\mathrm{KdV}$ equation with its solitary wave solutions. When both $\beta_{1}$ and $\gamma_{1}$ are finite, (1) does not admit steadily propagating, localized solitary wave solutions ${ }^{7}$. There does exist a family 
of steadily-translating periodic solutions that have the characteristics similar in some ways to the cnoidal wave solutions to the $\mathrm{KdV}$ equation, thus these periodic solutions to (1) have been termed quasi-cnoidal waves ${ }^{8,9}$. One particular approximate solution consists of sequence of KdV solitary waves joined by parabolic $\operatorname{arcs}^{10}$.

When a KdV solitary wave is used as the initial condition for (1), it will slowly decay due to radiation of inertia-gravity waves ${ }^{11-13}$. Furthermore, Grimshaw et al. ${ }^{12}$ used an asymptotic analysis to show that the radiation leads to a complete decay of the initial solitary wave in a finite time. Numerical solutions of (1) with small $\gamma_{1}$ (i.e. small rotation) are in good agreement with this theory ${ }^{12}$. With increased rotation, and a periodic domain, an interesting recurrence occurred. The initial solitary wave decayed by radiation; however, the radiated inertia-gravity wave, which was now larger amplitude, could itself steepen to produce a secondary solitary wave with an amplitude comparable to the initial wave ${ }^{11}$. This was followed by another episode of radiation decay, ultimately leading to a near-periodic recurrence of the solitary wave riding on a longer background inertia-gravity wave. A similar recurrence of the initial solitary wave was also found in an open system ${ }^{12}$. However, this phenomena was not explored in great detail (only one run is reported), nor were the numerical solutions carried to large times to see if the recurrence was indeed periodic.

The objective of this paper is to further examine the radiation decay of solitary waves and the recurrence phenomenon. One of the principal reasons for the failure of KdV-type models to accurately capture the details of observed wave evolution is that the amplitudes are frequently well beyond the weakly nonlinear realm. Thus, the restriction to weakly-nonlinear waves will be relaxed, while retaining the assumption of long, weakly nonhydrostatic waves. Numerical solutions to these new governing equations are then explored in the case of an initial solitary wave in an unbounded domain.

\section{THE MODEL}

The situation under consideration is an inviscid, two-layer fluid with layer depths $h_{i}$ and velocity vectors $\boldsymbol{u}_{i}$. Here $i=1$ and 2 refer to the upper and lower layers, respectively. The layer densities are $\rho_{1}$ and $\rho_{2}=\rho_{1}+\Delta \rho$. The system is rotating about the $z$-axis with constant Coriolis frequency $f(>0)$. The gravitational acceleration $g$ is directed in the negative $z$-direction. In the absence of motion $h_{1}=h_{0}$ and $h_{2}=H-h_{0}$, where $H$ is the 
total depth. The bottom is flat and the upper surface is rigid.

The propagation of nonlinear interfacial waves in this system will be studied in the limit of fully nonlinear, $\alpha=O(1)$, and weakly nonhydrostatic long waves, $\beta \ll 1$. In the absence of rotation Miyata ${ }^{14}$ and Choi and Camassa ${ }^{15}$ derived a coupled set of equations (denoted as the MCC equations for brevity) for fully nonlinear, weakly nonhydrostatic interfacial waves. The extension of the MCC theory to include rotation mirrors the non-rotating derivation of Choi and Camassa ${ }^{15}$, so only the additional details related to rotation are outlined here.

The waves will be taken to propagate in the $x$-direction. With rotation, motion in the transverse $y$-direction will occur; however, it will be assumed that $\partial / \partial y=0$. From Choi and Camassa ${ }^{15}$, the continuity and vertically averaged $x$-momentum equations for each layer, including the Coriolis term, are

$$
\begin{aligned}
h_{i t}+\left[h_{i} \bar{u}_{i}\right]_{x} & =0 \\
\bar{u}_{i t}+\bar{u}_{i} \bar{u}_{i x}-f \bar{v}_{i} & =-g \eta_{x}+\frac{1}{\rho_{i}} P_{x}+D_{i}+O\left(\beta^{2}\right) .
\end{aligned}
$$

Here the overbar indicates a vertical average over a layer, $u_{i}$ and $v_{i}$ are, respectively, the velocities in the $x$ and $y$-directions, $\eta(x, t)=h_{0}-h_{1}$ is the interface displacement, and $P(x, t)$ is the pressure at the interface. The subscripts $t$ and $x$ indicate differentiation. The $O(\beta)$ nonhydrostatic effects are given by ${ }^{15}$

$$
D_{i}=h_{i}^{-1}\left[\frac{1}{3} h_{i}^{2}\left(\bar{u}_{i x t}+\bar{u}_{i} \bar{u}_{i x x}-\left(\bar{u}_{i x}\right)^{2}\right)\right]_{x} .
$$

The exact, vertically averaged $y$-momentum equations are

$$
\left(h_{i} \bar{v}_{i}\right)_{t}+\left(h_{i} \overline{u_{i} v_{i}}\right)_{x}+f h_{i} \bar{u}_{i}=0
$$

When $u_{i}$ is expanded in powers of $\beta$,

$$
u_{i}=u_{i}^{(0)}+\beta u_{i}^{(1)}+O\left(\beta^{2}\right)
$$

it follows directly from the first-order version of (3) with $f=0$ that $u_{i}^{(0)}$ is independent of $z$ if $u_{i z}^{(0)}=0$ at $t=0$. With rotation, expansion of $v_{i}$, along with the condition that $v_{i z}^{(0)}=0$ at $t=0$, similarly gives $v_{i}^{(0)}=v_{i}^{(0)}(x, t)$. Thus $\overline{u_{i} v_{i}}=\bar{u}_{i} \bar{v}_{i}+O\left(\beta^{2}\right)$, and after using (2), (5) gives to $O\left(\beta^{2}\right)$,

$$
\bar{v}_{i t}+\bar{u}_{i} \bar{v}_{i x}+f \bar{u}_{i}=0
$$


Equations (2), (3), and (6) can be simplified by eliminating $P_{x}$. The Boussinesq approximation $\Delta \rho / \rho_{1} \ll 1$ is also employed since it is reasonable in the oceanic context and consistent with the rigid-lid assumption. First, though, the equations are nondimensionalized using $\sqrt{g^{\prime} H}, H, l$, and $l / \sqrt{g^{\prime} H}$ for $\left(\bar{u}_{i}, \bar{v}_{i}\right), h_{i}, x$, and $t$, respectively. The reduced gravity $g^{\prime}=g \Delta \rho / \rho_{1}$. The resulting nondimensional, rotating MCC equations (MCC-f) are

$$
\begin{aligned}
h_{1 t}+\left[s h_{1}\left(h_{1}-1\right)\right]_{x} & =0 \\
s_{t}+\left[\frac{1}{2} s^{2}\left(2 h_{1}-1\right)-h_{1}\right]_{x}-\gamma v & =\beta\left(D_{2}-D_{1}\right) \\
v_{t}+s v h_{1 x}+s\left(2 h_{1}-1\right) v_{x}+s V_{x}+\gamma s & =0 \\
V_{t}+\left[s v h_{1}\left(1-h_{1}\right)\right]_{x} & =0 .
\end{aligned}
$$

Here $s=\bar{u}_{2}-\bar{u}_{1}$ and $v=\bar{v}_{2}-\bar{v}_{1}$. Note that $\eta\left(=h_{0}-h_{1}\right)$ has been eliminated in favor of $h_{1}$ and $h_{2}=1-h_{1}$. The barotropic transports in the $x$ and $y$-directions are, respectively,

$$
U=\bar{u}_{1} h_{1}+\bar{u}_{2} h_{2}, \quad V=\bar{v}_{1} h_{1}+\bar{v}_{2} h_{2} .
$$

With the rigid-lid assumption $U=U(t)$ and must be specified. In (7)-(10) it has been set to $U=0$. The nonhydrostatic terms $D_{i}$ are given by (4) with $\bar{u}_{1}=s\left(h_{1}-1\right)$ and $\bar{u}_{2}=s h_{1}$.

The two parameters that appear in (7)-(10) are

$$
\gamma=\frac{l}{L_{R}}, \quad \beta=\left(\frac{H}{l}\right)^{2}
$$

where the scaling depth $h_{s}$ is set to $H$ in $\beta$. With this scaling $L_{R}=\sqrt{g^{\prime} H} / f$. As already introduced, these parameters measure the relative effects of rotation and non-hydrostatic dispersion, respectively. Note that unlike in (1), $\gamma$ is not restricted to be small. Equation (1) can be obtained from (7)-(10) in the small amplitude, weakly-rotating limit after restriction to unidirectional propagation.

When $\gamma=0$ with $\beta$ finite, and (7) and (8) reduce to the (non-rotating) MCC equations. If $v=V=0$ at $t=0$, they remain zero from (9) and (10). Solitary waves solutions can then be found from integration of ${ }^{14-16}$

$$
\left(\frac{d \eta}{d \zeta}\right)^{2}=\beta^{-1} 3 \eta^{2}\left[\frac{1-c^{-2}\left(h_{0}-\eta\right)\left(1-h_{0}+\eta\right)}{h_{0}^{2}\left(1-h_{0}+\eta\right)+\left(1-h_{0}\right)^{2}\left(h_{0}-\eta\right)}\right],
$$

where $\zeta=x-c t$,

$$
c= \pm\left[\left(h_{0}-\eta_{0}\right)\left(1-h_{0}+\eta_{0}\right)\right]^{1 / 2}
$$


is the phase speed, and $\eta_{0}$ is the wave amplitude. In the limit $\eta_{0} \rightarrow 0, c \rightarrow c_{0}=\left(h_{0}-h_{0}^{2}\right)^{1 / 2}$, the linear long wave phase speed in this two-layer system. The shear $s(\zeta)$ is given by

$$
s=\frac{c \eta}{\left(h_{0}-\eta\right)\left(1-h_{0}+\eta\right)} .
$$

As in weakly nonlinear theory, the solitary waves point into the deeper layer so that $\eta_{0}<0$ $(>0)$ for $h_{0}<0.5(>0.5)$. There are no solitary wave solutions for $h_{0}=0.5$. Solitary waves are limited to a maximum amplitude $\eta_{0 \max }=h_{0}-0.5$ that reaches mid-depth. This limiting wave has infinite wavelength and is a smooth, dissipationless transition between two uniform (conjugate) states. These "thick" solitary waves and conjugate state do not arise in the KdV equation, but do appear when the cubic nonlinearity is included in the KdV-type models ${ }^{17}$. The solitary waves from (13) agree quite well with full nonhydrostatic numerical calculations, laboratory experiments, and oceanic observations ${ }^{15,18-20}$.

The MCC equations, unlike their weakly nonlinear counterparts, do not filter out KelvinHelmholtz instability ${ }^{21}$. As a consequence, the solitary waves can be unstable at high wave numbers. Numerical solutions of the MCC equations show that if the grid resolution was too fine, unstable short waves first emerge near the wave crest and ultimately overwhelm the calculations ${ }^{21}$. This instability is enhanced for large amplitude waves, especially as $h_{0}$ becomes small (or approaches one). The instability can be controlled by filtering out wavenumbers above the linear stability threshold ${ }^{22}$.

In the limit $\beta=0$ with $\gamma$ finite, (7)-(10) are the shallow-water equations and have no solitary wave solutions. However, periodic, finite amplitude inertia-gravity wave solutions can be found ${ }^{23}$. As in (1) with $\beta_{1}=0$, these periodic waves arise from a balance between nonlinearity and low-frequency rotational dispersion. For a fixed frequency (or wavelength), these inertia-gravity waves exist up to a limiting amplitude, beyond which the rotational dispersion is insufficient to balance nonlinearity. These waves come in two classes. In the first, the limiting wave has a corner shape where the interface slope, $\eta_{x}$, is discontinuous at the wave crest. This solution is the fully-nonlinear analog of the sequence of parabolic arcs found for (1) with $\beta_{1}=0$. In the second type, the limiting wave has a lobe shape with $\eta_{x}=0$ at both crest and trough.

When both $\gamma$ and $\beta$ are finite it has not been possible to find steadily propagating solitary wave solutions. This is, of course, not too surprising since such solutions do not exist in the weakly nonlinear limit ${ }^{3,7}$. The question of periodic quasi-cnoidal solutions to the MCC-f 
equations is unresolved.

In the next section the evolution of MCC solitary waves (13) under the influence of rotation are considered. It is found that the solitary wave initially decays by radiation of inertia-gravity waves. The radiated long inertia-gravity wave always steepens, leading to the recurrence of a solitary-like wave. For long times, a nearly localized packet emerges. It consists of a long wave envelope and shorter, faster solitary-like waves that propagate through the envelope only to decay at the head and re-emerge at the tail.

\section{NUMERICAL SOLUTIONS}

\section{A. Methods}

The MCC-f equations, (7)-(10), are solved with the numerical method developed for a set of wave equations that are closely related to the non-rotating single-layer version of the MCC equations $^{24}$. The method uses centered, fourth-order finite differences for all $x$-derivatives except those in $D_{i}$, which are differenced using centered, second-order stencils. Temporal integration is a fourth-order Adams-Bashforth predictor-corrector scheme with iteration on the corrector step. Only minor modifications of the scheme are necessary for the MCC-f equations.

The solutions are initiated with a solitary wave, found from integration of (13), with $c>0$. To achieve long integration times without using an exceptionally large, or periodic, grid, the numerical domain is periodically extended in the positive $x$-direction (with $h=h_{0}$ and $s=v=V=0$ ). An equal number of points are removed from the upstream boundary. Some linear damping is added near this boundary to relax $s, v$, and $V$ toward zero and $h$ to $h_{0}$ and minimize any reflection from the grid shifting. The grid domain was large enough to minimize disturbances generated at moving upstream boundary affecting the leading part of the solution.

The scheme was successfully tested by checking solitary wave propagation in the absence of rotation. The solitary wave instability ${ }^{21}$ was found in some cases, and could be controlled by high-wavenumber filtering. However, in the results presented below this was not necessary. The instability was either not present, or controlled by using grid size with a maximum wavenumber below the stability threshold. In all cases a minimum of about 25 grid points 
per solitary wave are used.

To organize the presentation of results the lengthscale $l$ has been set to $L_{R}$ in (12), giving $\gamma=1$ and $\beta=\left(H / L_{R}\right)^{2}=H f^{2} / g^{\prime}$ in (7)-(10). This choice is arbitrary and it is possible to take $l=H$, giving $\beta=1$ and $\gamma=H / L_{R}$. In either case, the sole remaining parameter is $H / L_{R}$ and conversion between them amounts to a rescaling of $x$ and $t$. In the former scaling the inertia-gravity waves have lengths of $O(1)$ and the solitary waves are an orderof-magnitude shorter. Typical mid-latitude oceanic values of $H=100-4000 \mathrm{~m}$ (coastal to deep ocean), $f \approx 10^{-4} \mathrm{~s}^{-1}$, and $g^{\prime} \approx 0.03 \mathrm{~m} \mathrm{~s}^{-2}$ give $\beta \approx[0.3-13] \times 10^{-4}$. Thus, $\beta^{1 / 2}=H / L_{R}=0.01,0.02$ and 0.04 are investigated.

The other independent variables are $h_{0}$ and $\eta_{0}$. In order to minimize the emergence of the Kelvin-Helmholtz instability most results below are for $h_{0}=0.25$, though smaller values, with high-wavenumber filtering implemented as needed, have been investigated and similar results have been obtained. Wave amplitudes spanning the allowable range have been considered.

\section{B. Results}

Figure 1 shows an example with $\left[h_{0}, \beta^{1 / 2}\right]=[0.25,0.02]$. The initial solitary wave amplitude $\eta_{0}=-0.2$. The figure shows $\eta(x, t)$ in the frame moving with the linear phase speed $c_{0}$, at equally spaced time intervals. Initially, the solitary wave decays by radiation of long inertia-gravity waves. However, by $t \approx 10$, the inertia-gravity wave has steepened to produce a packet of short solitary-like waves. The largest, lead wave of this group quickly separates from the group and grows in amplitude. By the time this secondary wave has reached its maximum amplitude at $t \approx 30$, the primary wave amplitude has decayed by $\approx 80 \%$. This decay and re-emergence process repeats until a nearly localized, temporally

periodic wavepacket separates from the initial disturbances. This waveform consists of a long $O\left(L_{R}\right)$ envelope through which a shorter solitary-like waves propagate. The speed of the packet, or group speed, is just slightly greater than $c_{0}$.

The maximum interfacial displacement $|\eta|$ in this solution is shown in Fig. 2. Note that this figure shows times significantly greater than those shown in Fig. 1. Also shown is the 
solitary wave amplitude from weakly nonlinear asymptotic theory ${ }^{12}$

$$
\frac{\eta}{\eta_{0}}=\left(1-\frac{t}{t_{d}}\right)^{2}, \quad t_{d}^{2}=\frac{3 \eta_{0}\left(2 h_{0}-1\right)}{c_{0}^{2} \beta \gamma^{2}},
$$

where $t_{d}$ is the terminal damping time. In this example, the initial decay is given reasonably well by (16) even though the wave amplitude is well beyond the weakly nonlinear regime. For large times the maximum wave amplitude decays very slowly, but at $t>400$ it is still greater than $60 \%$ of the initial amplitude. The period between maximum amplitude peaks decreases until $t>240$ when it becomes nearly constant at 20.9.

The robust nature of the leading wavepacket is further emphasized when energy is considered. The MCC-f equations can be manipulated to obtain an energy equation (following Choi and Camassa ${ }^{15}$ ), where the energy [to $O(\beta)$ ] between $x=x_{1}$ and $x_{2}$ is

$$
E=\int_{x_{1}}^{x_{2}}\left[\frac{1}{2} \eta^{2}+\sum_{i=1}^{2} \frac{1}{2}\left(h_{i}\left(\bar{u}_{i}^{2}+\bar{v}_{i}^{2}\right)+\frac{1}{3} \beta h_{i}^{3} \bar{u}_{i x}^{2}\right)\right] d x
$$

The first term on the right is the potential energy from the interfacial displacement. The sum is kinetic energy integrated over the layers. The last term in the sum arises from the kinetic energy in the vertical velocity field $\overline{w_{i}^{2}} / 2$. Figure 3 shows $E$ integrated over a range of $x$ within 1.5 of the leading edge of the wavepacket in Fig. 1. It has been normalized by the energy of the initial solitary wave $E_{0}$. As does the maximum amplitude, the integrated energy decays rapidly until about $t=100$. It then decreases much more slowly, perhaps approaching a steady asymptotic value of $\sim 38.5 \%$ of the initial energy.

Both the evolution of the maximum amplitude and the energy of the leading wavepacket suggest a possible asymptotic approach to a localized state where the the radiation and energy loss to trailing inertia-gravity waves ceases. However, examination of the solutions indicates that decay and re-emergence process results in the continual weak radiation. Figure 4 shows a close-up of the leading wavepacket and the smaller trailing waves at $t=291.56$. However, these small amplitude waves drain very little energy (which goes as the square of the amplitude). While it appears that this leading wavepacket is not a completely localized structure, it is remarkably long lived. For reference, an inertial period is $2 \pi$.

Figures 5 and 6 show two more examples. In Fig. 5 the initial wave amplitude $\eta_{0}=-0.05$ is smaller than in Fig. 1 (where $\eta_{0}=-0.2$ ). The other parameters, $\left[h_{0}, \beta^{1 / 2}\right]=[0.25,0.02]$, are unchanged. The evolution for this smaller initial wave is much the same, with a rapid emergence of a localized wavepacket after the steepening of the radiated inertia-gravity wave. 
Again, a substantial fraction of the initial energy, now $\approx 48 \%$, is contained in the leading wavepacket at the last time shown. The packet speed is now slightly less than $c_{0}$ and the time between amplitude maxima is $\approx 26.3$.

In Fig. 6 both $\eta_{0}=-0.2$ and $h_{0}=0.25$ are the same as Fig. 1 ; however, $\beta^{1 / 2}=0.01$ is smaller by a factor of two. When compared to Fig. 1, the early evolution for $t<100$ is more complex. The development of the leading packet takes longer, and involves two separate steepening events in the radiated inertia-gravity wave train. Despite the complicated initial period a recurrent waveform does appear for $t>100$, though it is substantially weaker than the previous two examples. At $t=180$ only about $6 \%$ of the initial energy is contained in the leading packet. The time between amplitude maxima is $\approx 30.7$.

These results are typical of other values of $h_{0}, \beta$, and $\eta_{0}$. The time for the localized packet to emerge and its other characteristics depend on the parameters. Some of these characteristics are illustrated in Fig. 7 which shows the leading disturbances with $h_{0}=0.25$ for a set of runs in which $\beta$ and $\eta_{0}$ are varied. All the solutions are at times after the energy is decaying very slowly and at a phase of the recurrence cycle when the solitary-like wave is close to the mid-point of the packet and the amplitude is maximum. The top three plots show runs with $\eta_{0}=-0.2$ and $\beta^{1 / 2}=0.01,0.02$, and 0.04 . The bottom two are for $\eta_{0}=-0.1$ and -0.05 with $\beta^{1 / 2}=0.02$. The similar structure of the solutions for the various values of $\eta_{0}$ and $\beta$ is clear. The figure also shows that decreasing $\beta$ with $\eta_{0}$ fixed (or vice-versa) leads to a smaller amplitude, shorter packet. The second and third solutions (from the top of the figure) have nearly the same amplitude $\max |\eta|=0.127$ and 0.128 , respectively. Thus, for a fixed amplitude, the length of the structure increases with $\beta^{1 / 2}$.

Recall that $x$ is scaled with $L_{R}$. If instead, $x$ is scaled with $H, x \rightarrow x / \gamma$, with $\gamma=H / L_{R}$ and $\beta=1$. This alternate scaling, where the influence of rotation increases with $\gamma$, shows that the packet length compared to the depth $H$ decreases as $\gamma$ or the amplitude increases.

Given the robust nature of these wavepackets it is of interest to explore their interactions. Two examples are shown in Figs. 8 and 9. The first shows a calculation initiated with two well-separated solitary waves of amplitudes $\eta_{0}=-0.2$ and -0.05 , with $h_{0}=0.25$ and $\beta^{1 / 2}=0.02$. From Figs. 1 and 5 it is expected that each solitary wave will produce a packet and that the one from the larger wave will overtake the one from the smaller wave. This is indeed what happens, and remarkably, the packets appear to pass through one another with little adverse effect. Comparison of the larger leading wavepacket with the one 
from Fig. 1 shows very little difference between the two. The smaller second packet from is noticeably distorted as it propagates through the inertia-gravity waves radiated by the larger wavepacket, but is still identifiable.

The second example (Fig. 9) is identical to the first except the smaller of the two initial solitary waves has $\eta_{0}=-0.1$. Instead of the soliton-like interaction above, the two packets merge (each is identifiable at $t \approx 50$ ). The resulting packet is initially more complicated than the clean examples seen so far, but it does remain intact. At times greater than shown, the leading packet becomes organized and nearly periodic with two or three solitary-like waves (depending on the phase) in the packet. Figure 10 shows a close up of the solution at $t=415.69$. Note that the packet is now longer than the earlier "single-wave" case.

The longevity of these wave packets must involve a near-balance of nonlinearity with both rotational and nonhydrostatic dispersion, though the details are still to be established. Figures 9 and 10 show that packets with more than one solitary-like wave can exist and suggest that these packets might be interpreted as a modulated periodic wave. Waves of the quasi-cnoidal type may be the underlying "fast" wave.

As a simple test of this idea, the approximate quasi-cnoidal solution of (1) can be compared with the properties of the waves in Fig. 10. This solution consists of sequency of solitary waves joined by parabolic arcs and is given (in the present notation and nondimensionalization) by $^{10}$

$$
\eta=-\frac{\eta_{0}}{9}+\eta_{0} \operatorname{sech}^{2}\left(\frac{x-c t}{\Delta}\right)+\frac{\gamma^{2}}{18\left(2 h_{0}-1\right)}\left[\left(x-c t-\frac{\lambda}{2}\right)^{2}-\frac{\lambda^{2}}{12}\right] .
$$

The wavelength $\lambda$ and the phase speed $c$ are given by

$$
\lambda=18 \Delta, \quad c=c_{0}+\frac{\left(2 h_{0}-1\right) \eta_{0}}{3 c_{0}}+\frac{\gamma^{2} \lambda^{2}}{72 c_{0}} .
$$

Here $\eta_{0}$ is the amplitude of the KdV solitary wave that comprises the inner solution at the junction of the arcs and

$$
\Delta^{2}=\frac{4}{3} \frac{\beta c_{0}^{4}}{\left(2 h_{0}-1\right) \eta_{0}}
$$

The amplitude of the peak of the quasi-cnoidal wave, $\eta_{M}$, is related to $\eta_{0}$ through

$$
\eta_{M}=\frac{8}{9} \eta_{0}+\frac{4 \gamma^{2} \beta c_{0}^{4}}{\left(2 h_{0}-1\right)^{2} \eta_{0}}
$$

From Fig. $10 \eta_{M} \approx-0.1$. For the conditions of that run, $h_{0}=0.25, \gamma=1$, and $\beta^{1 / 2}=0.02$, this gives $\eta_{0}=-0.11$, and from (18), $\lambda=0.32$ and $c-c_{0}=0.046$. The wavelength from 
Fig. 10 is $\lambda \approx 0.37$ and $c-c_{0}=0.026$, determined from the speed of the central wave in the figure. Given the strong nonlinearity of the numerical solution and the assumption of weak nonlinearity of the theory, the agreement is reasonable. One effect of strong nonlinearity is to reduce the wave speed below that predicted by weakly nonlinear theory ${ }^{15}$. The discrepancy in wave speeds is consistent with this.

An additional comparison is found from the linear dispersion relation obtained from (7)-(10), $\omega^{2}=\left(\gamma^{2}+c_{0}^{2} k^{2}\right)\left(1+\frac{1}{3} \beta c_{0}^{2} k^{2}\right)^{-1}$. Linear waves have a maximum group speed, $c_{g}=\partial \omega / \partial k$, at finite wavenumber $k$. For $h_{0}=0.25, \gamma=1$, and $\beta^{1 / 2}=0.02$, the maximum $c_{g}=0.94 c_{0}$ at $k=16.28$. This is close to the packet speed $c_{g} \approx c_{0}$ and $k=17.0$, based on $\lambda=0.37$, from Fig. 10. It is consistent with the fact that a packet is the leading disturbance in all the numerical solutions.

This development of the leading wavepackets is not a consequence of the full nonlinearity of the MCC-f equations. Weakly nonlinear equations with errors $O\left(\alpha^{2}, \alpha \beta, \beta^{2}\right)$ obtained from the MCC-f equations (see the Appendix) give the same qualitative results. There are, of course, quantitative differences with the fully nonlinear solutions, particularly for large amplitudes. This is illustrated in Fig. 11 where the leading waveform from Figs. 1 and 5 are shown along with the corresponding solutions from the weakly nonlinear model. The latter were initiated with a non-rotating, weakly nonlinear solitary wave with the same amplitude as the MCC-f runs. For the small amplitude $\eta_{0}=-0.05$ the differences in the two solutions is minor (Fig. 11(a)). When $\eta_{0}=-0.2$ the differences are substantial (Fig. 11(b)); however, the qualitative character of the solution remains. With the fully nonlinear model the leading wavepacket has a larger amplitude, is faster, and contains a greater fraction of the initial energy. Also shown in the figure are the same calculations with the MCC-f model with the $O(\alpha \beta)$ nonlinear-dispersive terms removed (i.e., the same dispersion operator, $D_{2}-D_{1}=\frac{1}{3} \beta c_{0}^{2} s_{x x t}$, as the weakly nonlinear model). The agreement with the full MCC-f model is quite good, especially after such long times, implying that the nonlinear dispersion is of secondary importance when compared to higher-order nonlinear terms. A careful study of the limitations of the weakly nonlinear model, or the MCC-f model with linear dispersion, is worthwhile, but not attempted here. 


\section{DISCUSSION}

The role of rotation on internal solitary waves was explored using a fully-nonlinear, weakly nonhydrostatic theory for interfacial waves in a rotating, two-layer system. Numerical solutions show that an initial solitary wave will decay by radiation of an inertia-gravity wave. The radiated wave will itself steepen to eventually produce a second solitary wave that grows at the expense of the initial wave. The process will then repeat, producing a decay and rebirth recurrence cycle. While this initial stage has been identified in several previous numerical solutions of the weakly-nonlinear Ostrovsky equation (1), this study shows that ultimately a new, nearly localized wavepacket emerges. The waveform consists of a long $\mathrm{O}\left(L_{R}\right)$ envelope through which shorter, faster solitary-like waves propagate, vanishing as they reach the leading edge and re-emerging at the trailing edge. These packets are not completely localized, but the trailing radiated waves are very weak. Consequently, the energy in an established wavepacket, which can be as much as half of the energy of initial solitary wave, decays on a very long timescale.

These results suggest that the packets found in the numerical solutions are modulated fully-nonlinear versions of the quasi-cnoidal waves found for (1). However, it remains to be seen if the MCC-f equations actually possess quasi-cnoidal solutions, though it does seem likely that they do. Computation of the properties of any steadily propagating periodic solutions of (7)-(10) is a substantial numerical task that is now being addressed. An additional effort is necessary to uncover the detailed dynamics of the packets. Work in that direction is underway, but in the simpler context of weakly nonlinear waves where analytical progress is more likely possible.

The timescale for the packet to clearly emerge from the initial solitary wave was on the order of the decay time (16), which for the ocean parameters introduce in Section 2, is about $1-4$ days. The recurrence period is similar. These timescales are long, but given the distances internal solitary waves are known to propagate (e.g., $400 \mathrm{~km}$ over 3 days in the South China Sea), it is reasonable to expect that the effects discussed here could be important. For example, over long distances individual solitary-like are ephemeral, yet the packets transmit substantial energy at a speed given approximately by the linear longwave phase speed. Furthermore, the generation of the packets is not limited to the decay of an initial solitary wave. Other more general initial conditions (not shown) also give rise to 
these wavepackets. This is not surprising since in the absence of rotation, general initial conditions will usually produce solitary waves, and thus the localized packets when rotation is present. It does suggest that these wavepackets may be a fundamental part of long-range propagation if internal solitary waves in the ocean.

\section{Acknowledgments}

This work was supported by a Woods Hole Oceanographic Institution Mellon Independent Study Award and ONR Grant N000140610798.

\section{APPENDIX}

MCC-f equations (7)-(10) can be reduced to a set of equations for weakly nonlinear waves by expanding the dependent variables in powers of $\alpha$,

$$
\eta=\alpha \eta^{(1)}+\alpha^{2} \eta^{(2)}+\ldots
$$

Dropping terms of $O\left(\alpha^{2}, \alpha \beta, \beta^{2}\right)$ results in the weakly nonlinear equations

$$
\begin{aligned}
\eta_{t}+\sigma[s \eta]_{x}+c_{0}^{2} s_{x} & =0 \\
s_{t}+\sigma s s_{x}+\eta_{x}-\gamma v & =\frac{1}{3} \beta c_{0}^{2} s_{x x t} \\
v_{t}+\sigma s v_{x}+s V_{x}+\gamma s & =0 \\
V_{t}+c_{0}^{2}[s v]_{x} & =0
\end{aligned}
$$

where $\sigma=2 h_{0}-1$. With $V=0$ these equations are the same as derived by Gerkema ${ }^{25}$, after recognizing that here $s$ and $v$ involve layer-averaged velocities rather than velocities at the interface. The retention of $V$ is a consequence of not restricting rotational effects to be weak. Equation (1) can be obtained from these equations in the limit of weak rotation and unidirectional propagation.

Non-rotating $(\gamma=v=V=0)$ solitary waves solutions $\eta(x-c t), s(x-c t)$ are found by integrating

$$
\left(s_{x}\right)^{2}=\frac{6}{c_{0}^{2}}\left[\frac{s^{2}}{2}-\frac{\sigma s^{3}}{6 c}+\frac{c_{0}^{2} s}{c \sigma}+\frac{c_{0}^{2}}{\sigma^{2}} \ln \left(1-\frac{\sigma s}{c}\right)\right],
$$

with $\eta=c_{0}^{2} s(c-\sigma s)^{-1}$. The wave speed $c$ follows from setting $s_{x}=0$ with $s=s_{0}=$ $c \eta_{0}\left(c_{0}^{2}+\sigma \eta_{0}\right)^{-1}$. 
Non-rotating solitary wave solutions to the MCC-f equations (7) and (8) without the mixed nonlinear-dispersive terms (i.e., $D_{2}-D_{1}=\frac{1}{3} \beta c_{0}^{2} s_{x x t}$ ) are found by integrating

$$
\left(s_{x}\right)^{2}=\frac{6}{c_{0}^{2}}\left[\frac{s^{2}}{2}+\frac{s\left(h_{1}-h_{0}\right)}{c}+\frac{s^{3}\left(1-2 h_{1}\right)}{6 c}-I_{1}+\frac{c^{2}}{3} I_{2}\right]
$$

with (15) relating $s$ and $h_{1}=h_{0}-\eta$. The phase speed $c$ is found by setting $s_{x}=0$ with $\eta=\eta_{0}$ and $s=s_{0}$. Here

$$
\begin{aligned}
I_{1}= & \ln \left(\frac{h_{1}-1}{h_{0}-1}\right)+h_{0} \ln \left(\frac{h_{1}\left(h_{0}-1\right)}{\left(h_{1}-1\right) h_{0}}\right) \\
I_{2}= & 3 h_{0}\left(1+2 h_{0}^{2}-3 h_{0}\right) \ln \left(\frac{h_{1}\left(h_{0}-1\right)}{\left(h_{1}-1\right) h_{0}}\right)+3 h_{0}^{2}\left(1-h_{0}\right)\left(h^{-1}-h_{0}^{-1}\right) \\
& -\frac{1}{2} h_{0}^{3}\left(h_{1}^{-2}-h_{0}^{-2}\right)+3 h_{0}\left(2 h_{0}-h_{0}^{2}-1\right)\left(\left(h_{1}-1\right)^{-1}-\left(h_{0}-1\right)^{-1}\right) \\
& +\frac{1}{2}\left(h_{0}^{3}+3 h_{0}-3 h_{0}^{2}-1\right)\left(\left(h_{1}-1\right)^{-2}-\left(h_{0}-1\right)^{-2}\right) .
\end{aligned}
$$

\section{REFERENCES}

${ }^{1}$ L. Ostrovsky and Y. Stepanyants, "Do internal solitons exist in the ocean?," Rev. Geophysics 27, 293 (1989).

${ }^{2}$ K. R. Helfrich and W. K. Melville, "Long nonlinear internal waves," Ann. Rev. Fluid Mech. 38, 395 (2006).

${ }^{3}$ L. Ostrovsky, "Nonlinear internal waves in a rotating ocean," Oceanology 18, 119 (1978).

${ }^{4}$ R. H. J. Grimshaw, "Evolution equations for weakly nonlinear, long internal waves in a rotating fluid," Stud. Appl. Math. 73, 1 (1985).

${ }^{5}$ R. Grimshaw, L. Ostrovsky, V. I. Shrira, and Y. A. Stepanyants, "Long nonlinear surface and internal gravity waves in a rotating ocean," Surveys in Geophys. 19, 289 (1998). 
${ }^{6} \mathrm{Y}$. Stepanyants, "On stationary solutions of the reduced Ostrovsky equation: periodic waves, compactons and compound solitons," Chaos, Solitons and Fractals 28, 193 (2006).

${ }^{7}$ A. I. Leonov, "The effect of the earth's rotation on the propagation of weak nonlinear surface and internal long oceanic waves," Annals NY Acad. Sci. 373150 (1981).

${ }^{8}$ L. Ostrovsky and Y. A Stepanyants, "Nonlinear surface and internal waves in a rotating fluids," A. V. Gaponov-Grekhov, M. I. Rabinovich, and J. Engelgrecht, Nonlinear Waves 3. Proc of the Gorky School 1989 (IUTAM, Springer, 1990), pp. 106-128.

${ }^{9}$ J. P Boyd and G.-Y. Chen, "Five regimes of the quasi-cnoidal, steadily translating waves of the rotation-modified Korteweg-de Vries ("Ostrovsky") equation," Wave Motion 35, $141(2002)$.

${ }^{10}$ O. A. Gilman, R. Grimshaw, and Y. A. Stepanyants, "Approximate analytical and numerical solutions of the stationary Ostrovsky equation," Stud. Appl. Math. 95, 115 (1995).

${ }^{11}$ O. A. Gilman, R. Grimshaw, and Y. A. Stepanyants, "Dynamics of internal solitary waves in a rotating fluid," Dyn. Atmos. Oceans 23, 403 (1996).

${ }^{12}$ R. Grimshaw, J-M He, and L. Ostrovsky, "Terminal damping of a solitary wave due to radiation in rotational systems," Stud. Appl. Math 101, 197 (1998).

${ }^{13}$ W. K. Melville, G. G. Tomasson, and D. P. Renouard, "On the stability of Kelvin waves," J. Fluid Mech. 2061 (1989).

${ }^{14}$ M. Miyata, "Long internal waves of large amplitude," K. Horikawa and H. Maruo, Nonlinear Water Waves (IUTAM, Springer-Verlag, 1987), pp. 399-406.

${ }^{15}$ W. Choi and R. Camassa, "Fully nonlinear internal waves in a two-fluid system," 
J. Fluid Mech. 396, 1 (1999).

${ }^{16}$ M. Miyata, "Long internal waves of large amplitude," La Mer 23, 43 (1985).

${ }^{17}$ T. Kakutani and N. Yamasaki, "Solitary waves on a two-layer fluid," J. Phys. Soc. Japan 45, 674 (1978).

${ }^{18} \mathrm{H}$. Michallet and E. Barthelemy, "Experimental study of interfacial solitary waves," J. Fluid Mech. 366, 159 (1998).

${ }^{19}$ L. Ostrovsky and J. Grue, "Evolution equations for strongly nonlinear internal waves," Phys. Fluids 15, 2934 (2003).

${ }^{20}$ R. Camassa, W. Choi, H. Michallet, P.-O. Rusås, and J. Sveen, "On the realm of validity of strongly nonlinear asymptotic approximations for internal waves," J. Fluid Mech. 549, 1 (2006).

${ }^{21}$ T.-C. Jo and W. Choi, "Dynamics of strongly nonlinear internal solitary waves in shallow water," Stud. Appl. Math. 109, 205 (2002).

${ }^{22} \mathrm{~W}$. Choi, personal communication.

${ }^{23}$ R. Plougonven and V. Zeitlin, "On periodic inertia-gravity waves of finite amplitude propagating without change of form at sharp density-gradient interfaces in the rotating fluid," Phys. Lett. A. 314, 140 (2003).

${ }^{24}$ G. Wei, J. T. Kirby, S. T. Grilli, and R. Subramanya, "A fully nonlinear Boussinesq model for surface waves. part 1. highly nonlinear unsteady waves," J. Fluid Mech. 294, 71 (1995).

${ }^{25} \mathrm{~T}$. Gerkema, "A unified model for the generation and fission of internal tides in a rotating ocean," J. Mar. Res. 54, 421 (1996). 


\section{Figure Captions}

Fig. 1: Numerical solution for a solitary wave with $\eta_{0}=-0.2$ and $\left[h_{0}, \beta^{1 / 2}\right]=[0.25,0.02]$. The interface $\eta(x, t)$ is shown at equal time intervals in a frame moving with the linear wave speed $c_{0}$.

Fig. 2: The maximum amplitude, $\max |\eta|$, of the solution in figure 1 is shown as a function of $t$ by the small circles. The dashed line is the amplitude decay from weakly nonlinear theory (16).

Fig. 3: The energy $E$ within $x=1.5$ of the leading edge of the leading wavepacket, normalized by the energy, $E_{0}$, of the initial solitary wave in Fig. 1.

Fig. 4: The interface displacement of the leading wavepacket from the run in Fig. 1 at $t=291.56$.

Fig. 5: Numerical solution for a solitary wave with $\eta_{0}=-0.05$ and $\left[h_{0}, \beta^{1 / 2}\right]=$ $[0.25,0.02]$. The interface $\eta(x, t)$ is shown at equal time intervals in a frame moving with the linear wave speed $c_{0}$.

Fig. 6: Numerical solution for a solitary wave with $\eta_{0}=-0.2$ and $\left[h_{0}, \beta^{1 / 2}\right]=$ $[0.25,0.01]$. The interface $\eta(x, t)$ is shown at equal time intervals in a frame moving with the linear wave speed $c_{0}$.

Fig. 7: The interface displacement $\eta$ of the leading wavepacket for $h_{0}=0.25$ with the parameters $\eta_{0}$ and $\beta^{1 / 2}$ indicated. Each plot is offset vertically by 0.1 and shown at a point in the calculation when the solitary-like wave is near the mid-point of the disturbance.

Fig. 8: Solution with two solitary waves, $\eta_{0}=-0.2$ and -0.05 , and $\left[h_{0}, \beta^{1 / 2}\right]=$ $[0.25,0.02]$. The interface $\eta(x, t)$ is shown at equal time intervals in a frame moving with the linear wave speed $c_{0}$. 
Fig. 9: Solution with two solitary waves, $\eta_{0}=-0.2$ and -0.1 , and $\left[h_{0}, \beta^{1 / 2}\right]=$ $[0.25,0.02]$. The interface $\eta(x, t)$ is shown at equal time intervals in a frame moving with the linear wave speed $c_{0}$.

Fig. 10: The interface displacement of the leading wavepacket from the run in Fig. 9 at $t=415.69$.

Fig. 11: Comparison of the leading waveform from the MCC-f model (solid), the weakly nonlinear model (dashed), and the MCC-f model with the linear non-hydrostatic dispersion (dash-dot). (a) $\left[h_{0}, \beta^{1 / 2}\right]=[0.25,0.02]$ with $\eta_{0}=-0.05$ at $t=144.34$ (b) The same for $\eta_{0}=-0.2$ at $t=153.96$. 


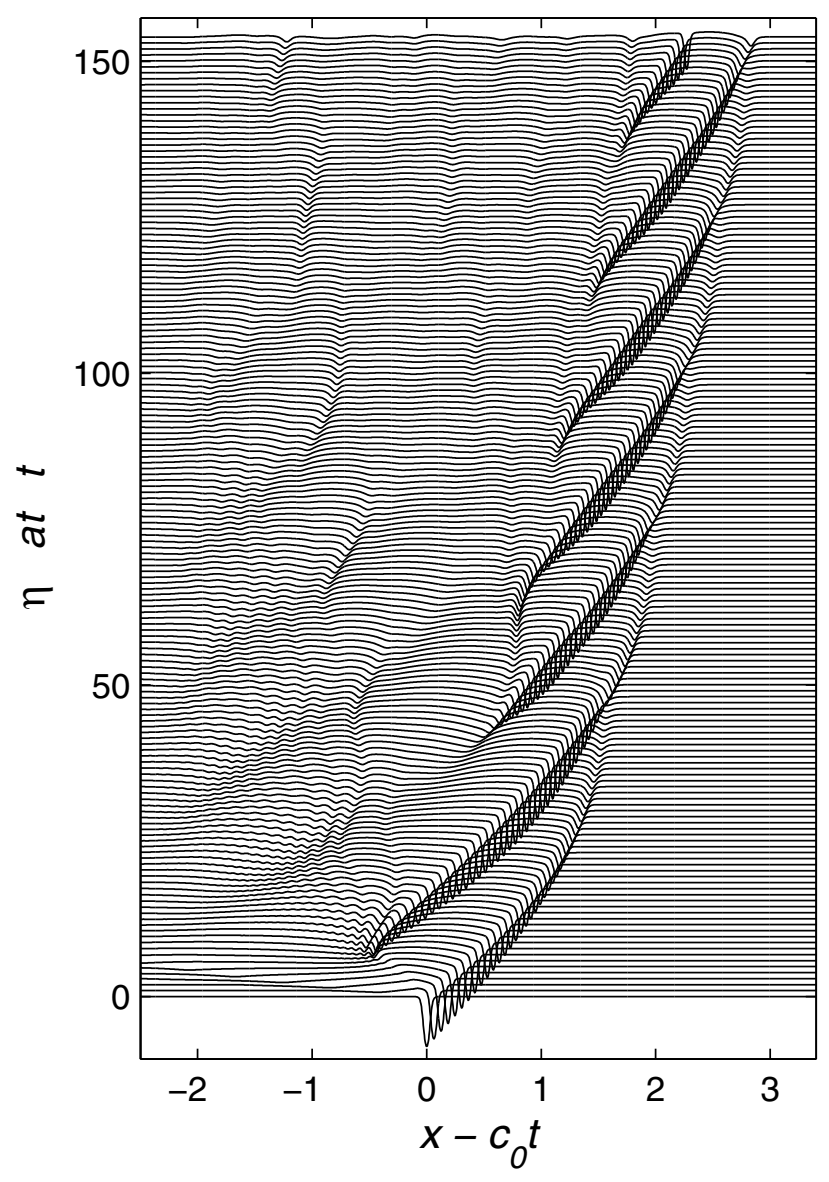

FIG. 1: Numerical solution for a solitary wave with $\eta_{0}=-0.2$ and $\left[h_{0}, \beta^{1 / 2}\right]=[0.25,0.02]$. The interface $\eta(x, t)$ is shown at equal time intervals in a frame moving with the linear wave speed $c_{0}$.

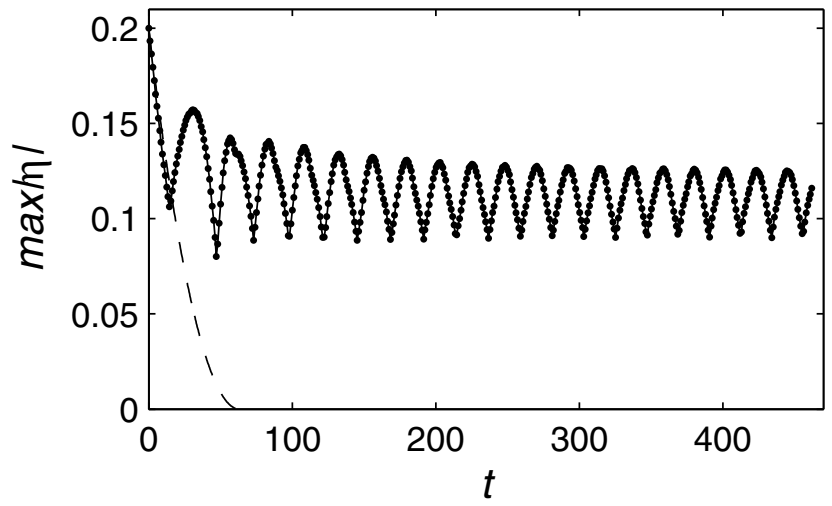

FIG. 2: The maximum amplitude, $\max |\eta|$, of the solution in figure 1 is shown as a function of $t$ by the small circles. The dashed line is the amplitude decay from weakly nonlinear theory (16). 


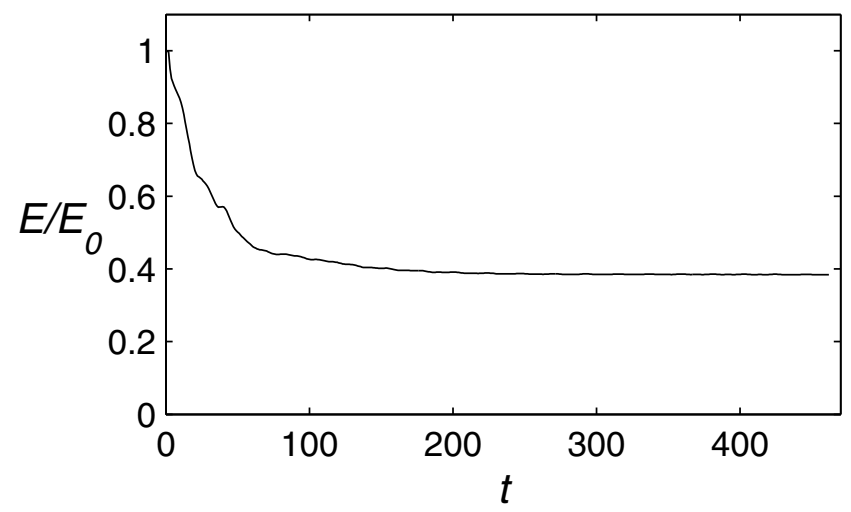

FIG. 3: The energy $E$ within $x=1.5$ of the leading edge of the leading wavepacket, normalized by the energy, $E_{0}$, of the initial solitary wave in Fig. 1.

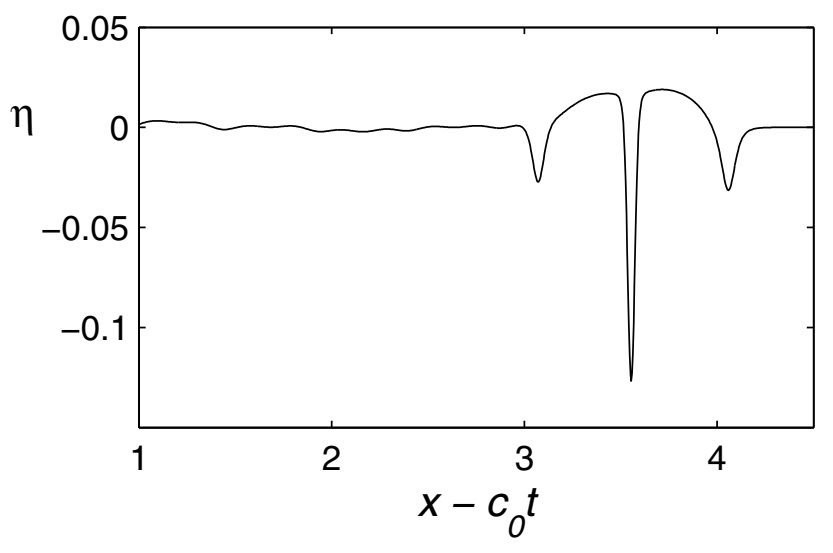

FIG. 4: The interface displacement of the leading wavepacket from the run in Fig. 1 at $t=291.56$. 


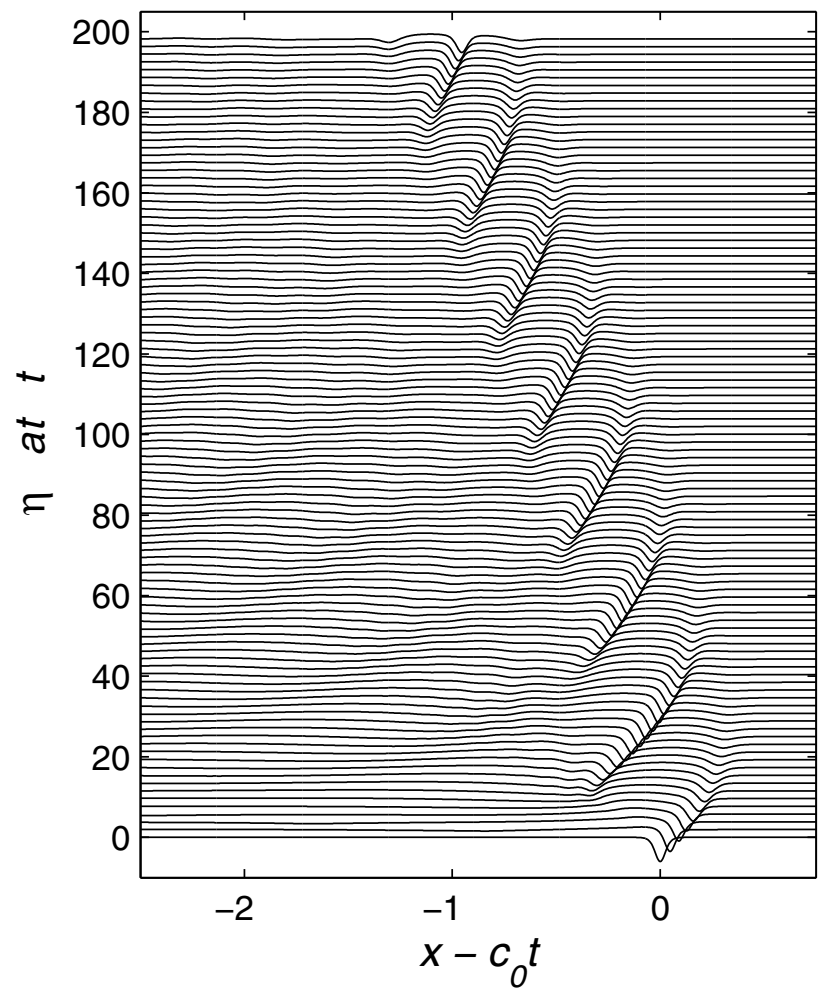

FIG. 5: Numerical solution for a solitary wave with $\eta_{0}=-0.05$ and $\left[h_{0}, \beta^{1 / 2}\right]=[0.25,0.02]$. The interface $\eta(x, t)$ is shown at equal time intervals in a frame moving with the linear wave speed $c_{0}$. 


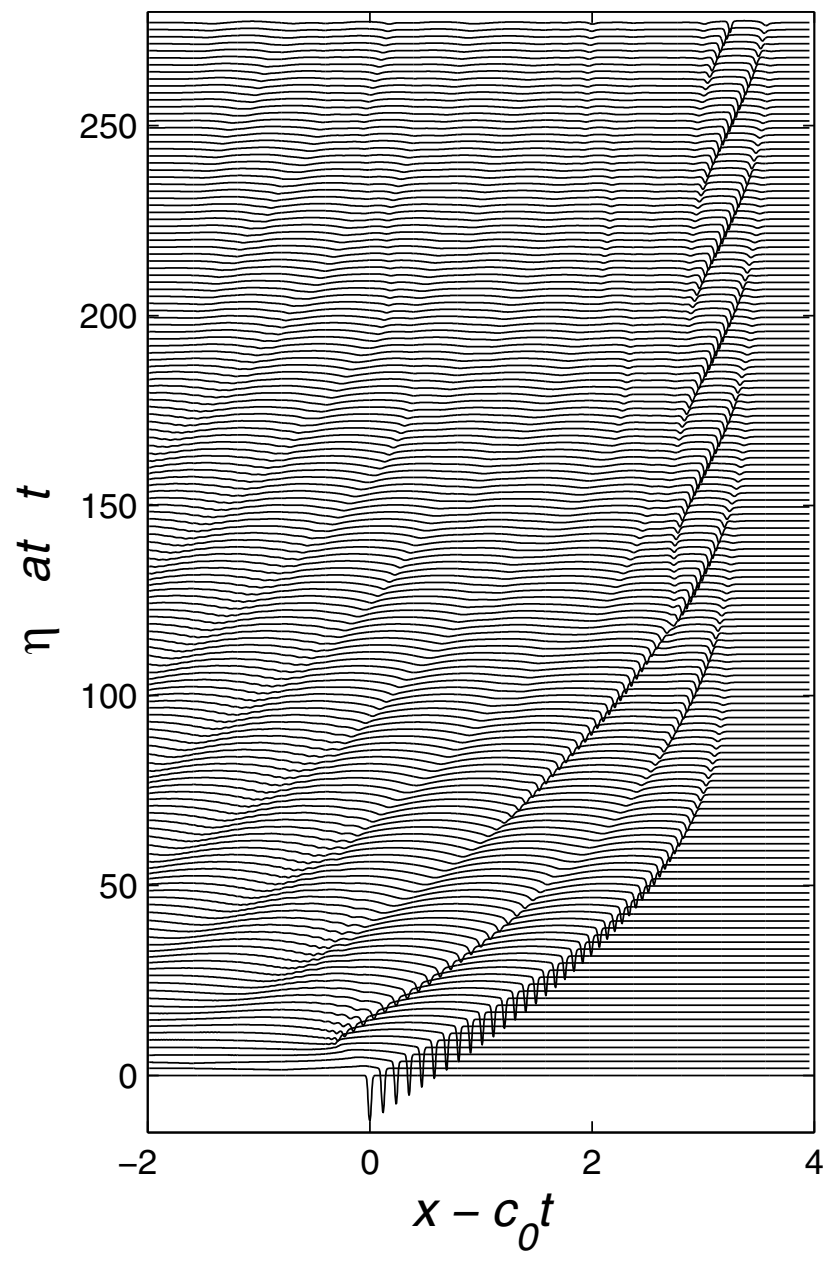

FIG. 6: Numerical solution for a solitary wave with $\eta_{0}=-0.2$ and $\left[h_{0}, \beta^{1 / 2}\right]=[0.25,0.01]$. The interface $\eta(x, t)$ is shown at equal time intervals in a frame moving with the linear wave speed $c_{0}$. 


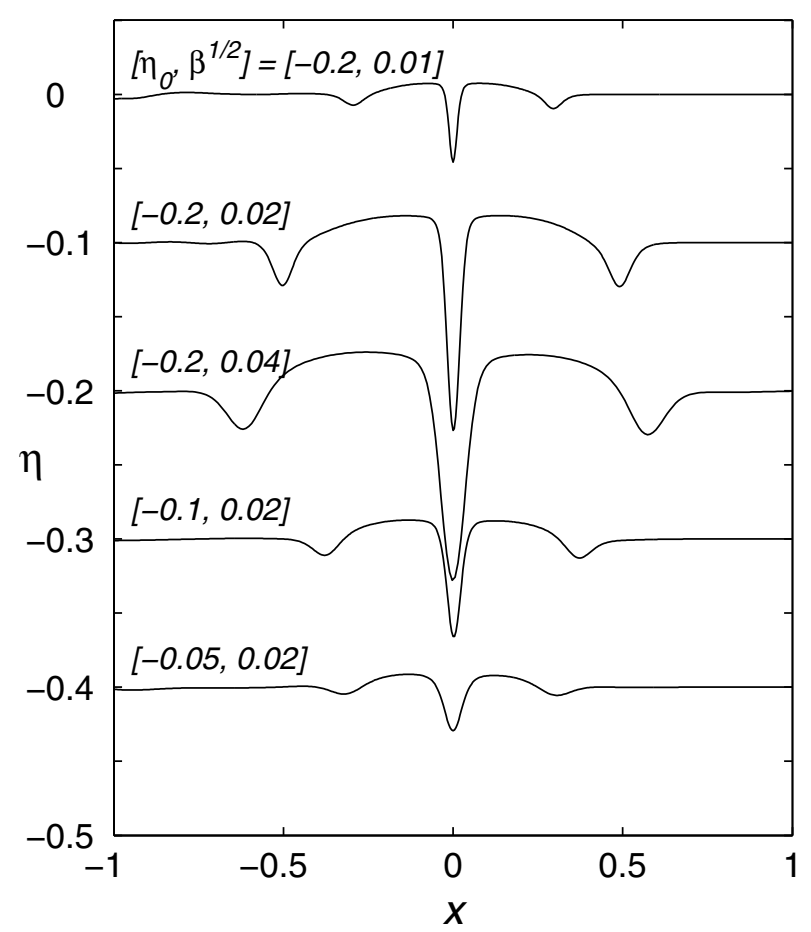

FIG. 7: The interface displacement $\eta$ of the leading wavepacket for $h_{0}=0.25$ with the parameters $\eta_{0}$ and $\beta^{1 / 2}$ indicated. Each plot is offset vertically by 0.1 and shown at a point in the calculation when the solitary-like wave is near the mid-point of the disturbance. 


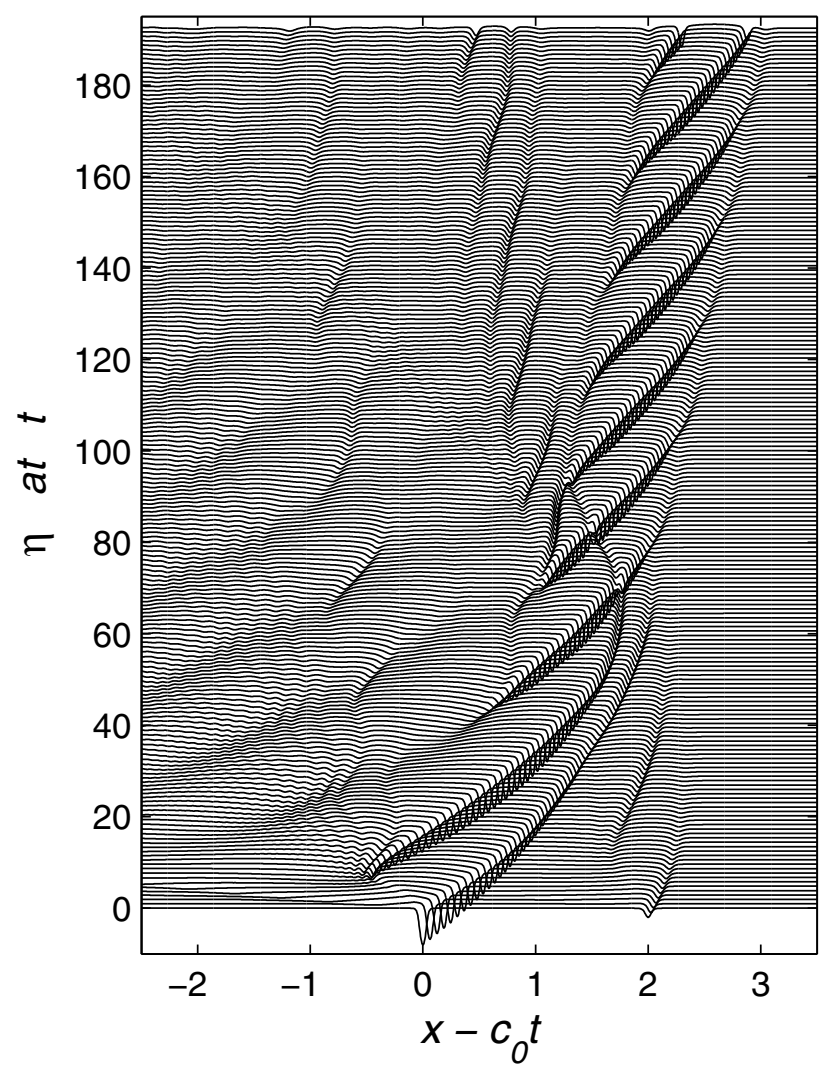

FIG. 8: Solution with two solitary waves, $\eta_{0}=-0.2$ and -0.05 , and $\left[h_{0}, \beta^{1 / 2}\right]=[0.25,0.02]$. The interface $\eta(x, t)$ is shown at equal time intervals in a frame moving with the linear wave speed $c_{0}$. 


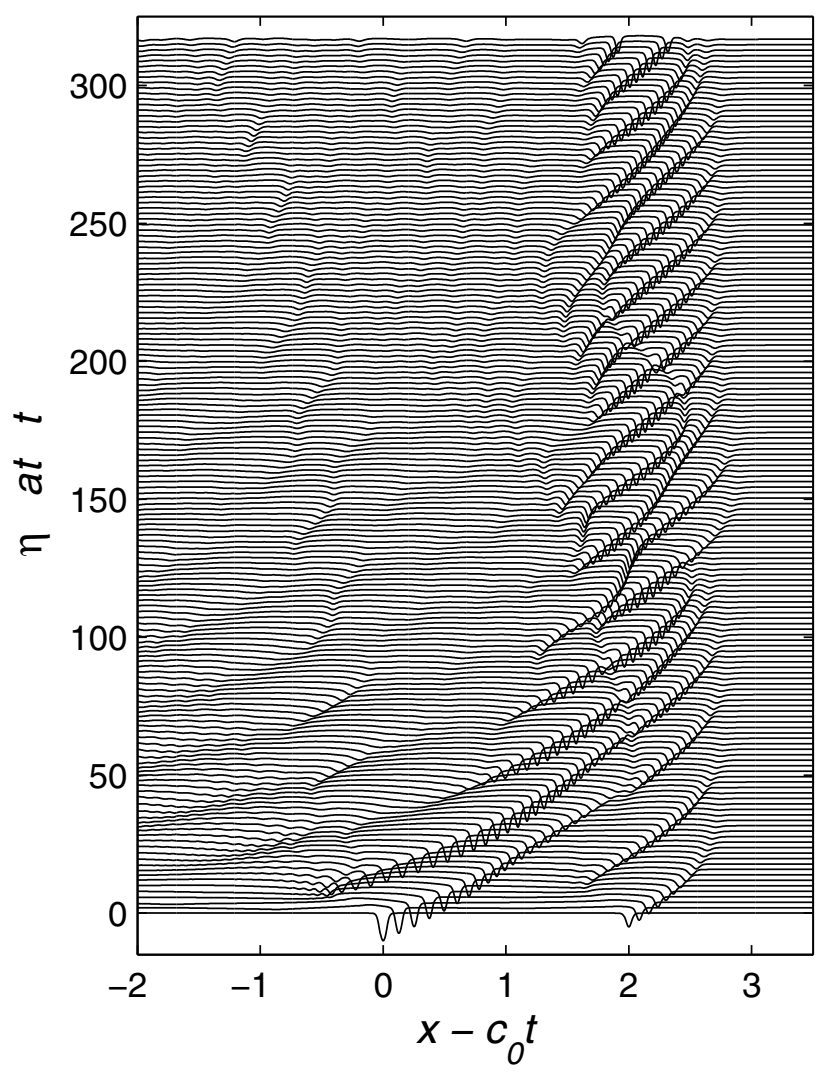

FIG. 9: Solution with two solitary waves, $\eta_{0}=-0.2$ and -0.1 , and $\left[h_{0}, \beta^{1 / 2}\right]=[0.25,0.02]$. The interface $\eta(x, t)$ is shown at equal time intervals in a frame moving with the linear wave speed $c_{0}$.

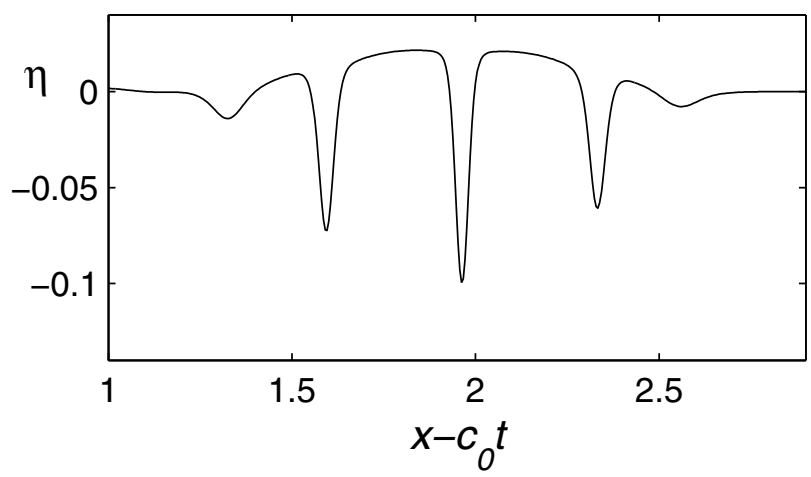

FIG. 10: The interface displacement of the leading wavepacket from the run in Fig. 9 at $t=415.69$. 

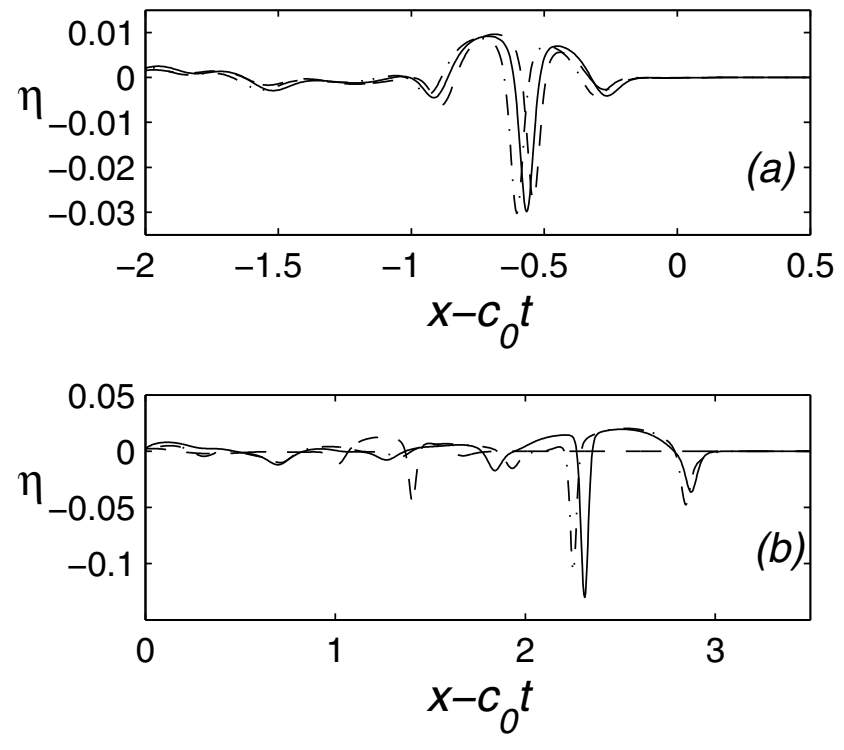

FIG. 11: Comparison of the leading waveform from the MCC-f model (solid), the weakly nonlinear model (dashed), and the MCC-f model with the linear non-hydrostatic dispersion (dash-dot). (a) $\left[h_{0}, \beta^{1 / 2}\right]=[0.25,0.02]$ with $\eta_{0}=-0.05$ at $t=144.34$ (b) The same for $\eta_{0}=-0.2$ at $t=153.96$. 